\title{
Commitment during Stenotele Differentiation in Hydra Is Localized Near the S/G2 Boundary in the Terminal Cell Cycle
}

\author{
Toshitaka Fujisawa ${ }^{1}$ and Charles N. David ${ }^{2}$ \\ Department of Molecular Biology, Albert Einstein College of Medicine, Bronx, New York 10461 \\ Received January 26, 1982; accepted in revised form May 4, 1982
}

\begin{abstract}
The timing of commitment during stenotele differentiation in Hydra was determined. Regeneration of isolated distal regions of the body column induces stenotele differentiation. The kinetics of appearance of committed stenotele precursors was determined in such regenerating pieces. Using $\left[{ }^{3} \mathrm{H}\right]$ thymidine labeling and hydroxyurea sensitivity, the G1/S and the S/G2 boundaries of the precursor population was also determined. Comparison of these results indicates that stenotele commitment is localized near the $\mathrm{S} / \mathrm{G} 2$ boundary in the terminal cell cycle of nests of precursor cells.
\end{abstract}

\section{INTRODUCTION}

Interstitial stem cells of Hydra give rise to four types of nematocytes: stenotele, desmoneme, holotrichous isorhiza, and atrichous isorhiza (Lehn, 1951; David and Gierer, 1974). Stem cells entering the nematocyte pathway undergo two to five rounds of synchronous cell division (Lehn, 1951; Rich and Tardent, 1969; David and Gierer, 1974) during which daughter cells remain connected to each other by cytoplasmic bridges to form nests of 4, 8, 16, and 32 cells (Slautterback and Fawcett, 1959). During the terminal cell cycle, all cells in a nest become committed to differentiate a specific capsule type (Fujisawa and David, 1981) and, following mitosis, capsule differentiation occurs over a 48- to 60-hr period depending on capsule type (David and Gierer, 1974). After completion of capsule differentiation, nests break up and individual nematocytes migrate into the tentacles. In Hydra attenuata used in the present study about $90 \%$ of all nematocytes are stenoteles and desmonemes.

The type of nematocyte differentiation depends on the position along the body column (Bode and Smith, 1977). Desmonemes differentiate mainly in the distal half of the body column, while stenoteles differentiate predominantly in the proximal half. As a consequence of this positional dependence, when distal tissue is isolated and allowed to regenerate a whole hydra it shifts from primarily desmoneme differentiation to both desmoneme and stenotele differentiation. In the present report we have investigated this induction of stenotele differentiation in order to localize more precisely the

\footnotetext{
1 Present address: Department of Biochemical Genetics, National Institute of Genetics, Mishima, Shizuoka 411, Japan.

${ }^{2}$ Present address: Zoologisches Institut, Universität München, Luisenstr. 14, 8000 Münich 2, West Germany.
}

time of stenotele commitment during the terminal cell cycle. Our results indicate that stenotele commitment occurs near the boundary between S and G2.

\section{MATERIALS AND METHODS}

\section{Hydra}

Hydra attenuata were cultured at $19^{\circ} \mathrm{C}$ in the medium containing $1 \mathrm{mM} \mathrm{CaCl}, 1 \mathrm{mM} \mathrm{NaHCO}$, and $0.01 \mathrm{~m} M$ EDTA. Animals were fed daily with freshly hatched Artemia salinas nauplii and washed $6 \mathrm{hr}$ later. Animals which had protrusions of their first buds were used for all experiments. The distal one-fifth of the body column was isolated and allowed to regenerate as described. Regenerating pieces were fed daily.

\section{Cell Type Analysis}

Differentiating stenoteles were scored in macerations using phase-contrast optics (David, 1973). Stenotele nematocytes differentiate in nests of 8 or 16 cells which break up into individual cells following maturation of the capsule. There are several recognizable stages in capsule differentiation. Initially the capsule is a rigid empty shell which appears gray in phase optics. Next the shell fills with refractile material and appears homogeneous yellow in phase optics. Finally, internal structures appear within the yellow background at which point it is no longer possible to distinguish differentiating and mature stenoteles based on capsule morphology. Such cells can only be identified as differentiating if they still occur in nests.

In the present experiments we have scored as "differentiating stenoteles" all cells which have a clearly defined but immature capsule (gray or homogeneous yellow in phase contrast) and cells with a more mature 


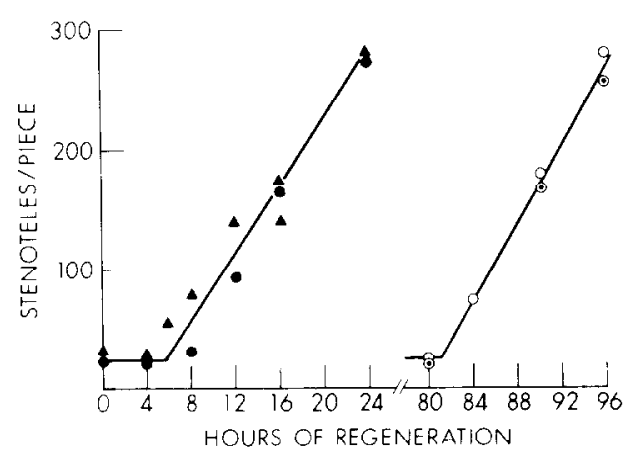

FIG. 1. Stenotele commitment and differentiation during regeneration of isolated distal pieces. Distal fifths of the body column were isolated and allowed to regenerate into whole hydra. The number of differentiating stenoteles was scored by maceration starting from 80 $\mathrm{hr}$ of regeneration (open symbols). Stenotele commitment was assayed by the scheme shown in Fig. 2 (closed symbols). Ten animals or pieces were macerated together for each point. Different symbols represent independent experiments.

capsule (internal structures present) if they still occur in nests (i.e., more than two cells attached to each other). Using these criteria, we find 360 differentiating stenoteles per hydra or 0.02 stenoteles per epithelial cell. This value agrees well with estimates of differentiating stenoteles made on the same animals by thiolacetic acid staining (David and Challoner, 1974). Thus our scoring procedure appears to identify most differentiating stenoteles.

\section{Labeling of Cells and Autoradiography}

$H y d r a$ were labeled with $\left[{ }^{3} \mathrm{H}\right]$ thymidine $(100 \mu \mathrm{Ci} / \mathrm{ml})$ and were subjected to autoradiography as described previously (Fujisawa and David, 1981).

\section{Vital Staining with Evans Blue}

Hydra were vitally stained with Evans blue (Wilby and Webster, 1970). The dye $(0.2 \mathrm{~g} /$ liter of culture solution) was injected directly into the gastric cavity one to two times a day for at least 3 days before experiments. The dye persists in digestive cells for at least 4 days.

\section{RESULTS}

\section{Stenotele Differentiation Induced by Regeneration of Isolated Distal Pieces}

When the distal one-fifth of the body column is isolated and allowed to regenerate into a whole hydra, cells in the distal region are effectively transplanted into all positions of the body column (Fujisawa and David, 1981). Since the rate of stenotele differentiation is low in distal tissue and high in proximal tissue (Bode and Smith, 1977), regeneration of isolated distal pieces in- duces stenotele differentiation. The appearance of newly differentiated stenoteles during regeneration is shown in Fig. 1. By 80-84 hr of regeneration, new stenoteles begin to appear and by $96 \mathrm{hr}$ their number has increased to 250 per regenerate from a level of about 20 in freshly isolated distal tissue.

The increase in Fig. 1 is preceded by an increase in commitment of precursors to the stenotele pathway. We have shown elsewhere that stenotele commitment occurs in the terminal cell cycle of precursor nests prior to the onset of capsule differentiation (Fujisawa and David, 1981). In order to define the appearance of committed stenoteles more precisely we have used the assay for committed stenoteles shown in Fig. 2. Distal fifths were isolated and allowed to regenerate. After varying periods of regeneration, pieces were grafted back onto an Evans blue-stained body column. Ninety-six hours after the start of regeneration, the unstained distal portion was excised, macerated, and scored for differentiating stenoteles. Two aspects of the procedure deserve special mention: (1) Stenoteles which are migrating from the proximal piece through the distal piece are not counted since our counting procedure does not score mature migrating stenoteles. (2) Induction of lateral basal disks at the site of regrafting does not occur since the regenerating tip is removed in the regraft procedure.

The procedure outlined in Fig. 2 tests the ability of stenotele precursors to differentiate when returned to a position in the body column which does not favor stenotele differentiation. We define this capacity for autonomous differentiation as commitment and use the procedure outlined in Fig. 2 to assay the appearance of committed stenotele precursors.

Figure 1 shows the appearance of committed stenoteles during regeneration of isolated distal pieces. Dur-

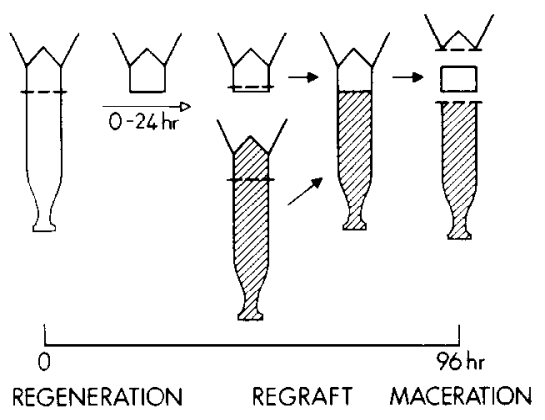

FIG. 2. Schematic representation of assay for stenotele commitment. Distal fifths of the body column were excised and allowed to regenerate. After varying periods of regeneration (0-24 hr) distal pieces were grafted back onto an Evans blue-stained body column. Ninety-six hours after the start of regeneration, the unstained distal portion was excised, macerated, and scored for differentiating stenoteles. 
ing the first $4 \mathrm{hr}$ of regeneration, there is no detectable increase in stenotele commitment. Thereafter stenotele commitment increases markedly. By $24 \mathrm{hr}$, regenerating pieces contain about 250 committed stenotele precursors compared to a level of $<20$ in equivalent nonregenerating tissue.

The curve in Fig. 1 indicates the true appearance of committed precursors only if the regrafting procedure leads to an immediate cessation of stenotele commitment. If there is a significant delay between the time of regrafting and the effective inhibition of commitment, then the measured number of stenoteles at any point will be higher than the number actually present at the time of regrafting. In this case the measured commitment curves precedes the true appearance of committed stenoteles by a time equal to the delay between regrafting and effective inhibition. In order to estimate the extent of this delay we performed an additional experiment to estimate the extent of commitment after regrafting.

Distal pieces were isolated and allowed to regenerate for $16 \mathrm{hr}$ before being regrafted to proximal pieces (see Fig. 2). This procedure induces commitment of a block of about 150 stenoteles over a 10-hr period from 6 to $16 \mathrm{hr}$. Beginning at $84 \mathrm{hr}$ the distal pieces were excised from the grafts, macerated, and scored for the appearance of differentiating stenoteles derived from the block of committed precursors. The results in Fig. 3 show the appearance of these cells between 88 and $96 \mathrm{hr}$ at the same rate as in Fig. 1. (The curve is delayed in this experiment by about $8 \mathrm{hr}$ compared to Fig. 1.) After 96 $\mathrm{hr}$ the rate of increase slows markedly and between 100 and $104 \mathrm{hr}$ the block of committed precursors completes differentiation and migrates out of the distal piece. Since the duration of the differentiated stage scored in these experiments is about $10 \mathrm{hr}$ (Fujisawa and David, 1981), the decrease in stenoteles between 100 and 104 hr strongly supports the idea that regrafting causes a

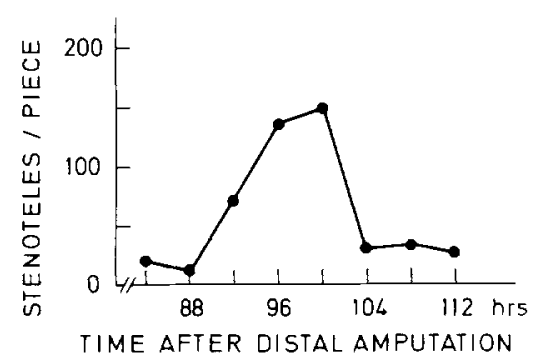

FIG. 3. Inhibition of stenotele commitment following regrafting. Distal fifths of the body column were allowed to regenerate for $16 \mathrm{hr}$ and then regrafted to Evans blue-stained body columns. From 84 to $112 \mathrm{hr}$ distal pieces were isolated from the grafts (see Fig. 2), macerated, and scored for differentiating stenoteles. Ten pieces were macerated for each point.

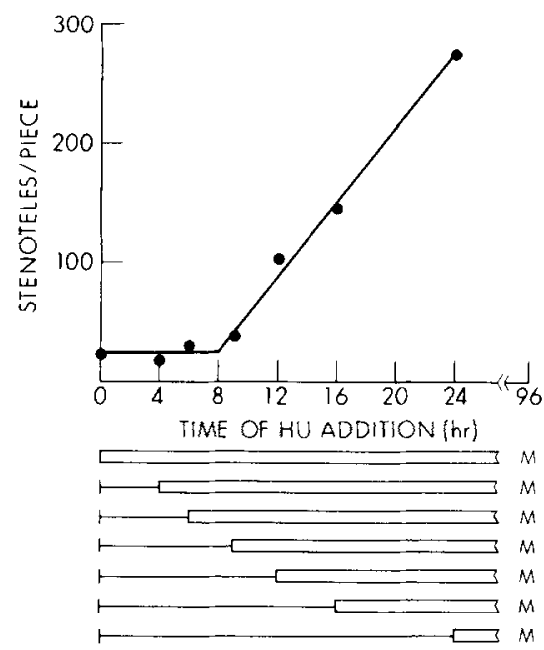

FIG. 4. Localization of the S/G2 boundary. The experimental procedure is outlined schematically in the bottom half of the figure. Distal fifths of the body column were isolated and allowed to regenerate. At various times from 0 to $24 \mathrm{hr}$ pieces were transferred to a medium containing hydroxyurea (HU) $(0.01 M)$ (open bars) and allowed to continue regeneration until $96 \mathrm{hr}$ after isolation. Hydroxyurea treatment was continued for $48 \mathrm{hr}$; thereafter animals were kept in hydroxyurea-free medium to avoid depression due to prolonged treatment. After $96 \mathrm{hr}$ of regeneration all pieces were macerated (M) and the number of differentiating stenoteles was scored. Results are plotled at the time HU treatment was initiated.

rapid inhibition of commitment. Otherwise differentiating stenoteles would persist in distal pieces for a longer period of time. Although it is not possible to define the kineties of inhibition precisely from the results in Fig. 3, it seems clear the inhibition is completed within 1-2 hr after regrafting. Thus the measured commitment curve (Fig. 1) closely approximates the true appearance of committed stenoteles in regenerating distal pieces.

\section{Localization of the $S / G 2$ Boundary}

The S/G2 boundary of the stenotele precursor population was determined using a procedure which tested the resistance of the precursor population to hydroxyurea (Fig. 4). Distal fifths of the body column were isolated and allowed to regenerate. At various times from 0 to $24 \mathrm{hr}$ regenerating pieces were transferred to medium containing hydroxyurea $(0.01 M)$ and allowed to continue regeneration. After $96 \mathrm{hr}$ of regeneration all pieces were macerated and the number of stenoteles was scored. The results of such an experiment are shown in Fig. 4. Addition of hydroxyurea beginning any time from 0 to $9 \mathrm{hr}$ completely inhibited stenotele differentiation; thereafter progressively later addition of hydroxyurea permitted a progressively larger fraction of stenotele precursors to differentiate. Since the major effect of hydroxyurea is to block cells in $\mathrm{S}$ phase, these 


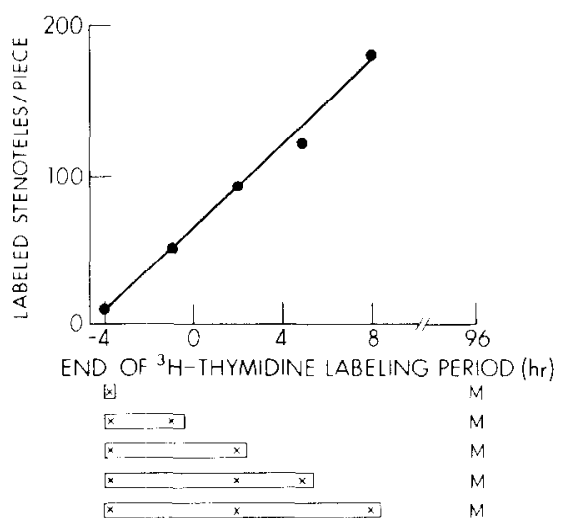

FIG. 5. Localization of the G1/S boundary. The experimental procedure is shown in the bottom half of the figure. Animals were labeled with $\left[{ }^{3} \mathrm{H}\right]$ thymidine (open bars) for inereasing lengths of time starting $4 \mathrm{hr}$ before isolation of distal pieces. Crosses in the bars indicate the time of $\left[{ }^{3} \mathrm{H}\right]$ thymidine pulses. At $96 \mathrm{hr}$ of regeneration pieces were macerated (M), autoradiographed, and scored for labeled stenoteles. Results are plotted at the end of labeling period.

results indicate that during the first $9 \mathrm{hr}$ of regeneration stenotele precursors are still in $\mathrm{S}$ phase. Beginning after $9 \mathrm{hr}$ increasing proportions of stenotele precursors pass from $\mathrm{S}$ into $\mathrm{G} 2$ at which point their differentiation is no longer blocked by hydroxyurea treatment. Thus the increase in "hydroxyurea-resistant stenotele differentiation" describes the kinetics with which the stenotele precursor population progresses from $S$ into G2. This is the S/G2 boundary of the precursor population.

\section{Localization of the G1/S Boundary}

The G1/S boundary of stenotele precursor population was determined by the procedure outlined in Fig. 5 (Venugopal and David, 1981). Animals were labeled with $\left[{ }^{3} \mathrm{H}\right]$ thymidine for increasing lengths of time starting $4 \mathrm{hr}$ before isolation of distal pieces. A single pulse of $\left[{ }^{3} \mathrm{H}\right]$ thymidine labels all cells in $\mathrm{S}$ phase at the time of the pulse. Repeated pulses label additional cells which enter $\mathrm{S}$ phase during the interval between pulses. Since S phase is $12 \mathrm{hr}$ long (David and Campbell, 1972; Campbell and David, 1974), pulses repeated at less than a 12-hr interval label all cells in $\mathrm{S}$ at least once. Thus the procedure outlined in Fig. 5 is equivalent to continuous labeling over the interval between pulses since all cells that are in $S$ phase or that enter $S$ phase during the labeling interval are labeled.

Figure 5 shows the results of such an experiment. When animals were given a single pulse at $-4 \mathrm{hr}$, no labeled stenoteles were observed at $96 \mathrm{hr}$. However, when the labeling period was extended from -4 to -1 $\mathrm{hr}$, the number of labeled stenoteles increased substantially. Thus precursors which entered S phase between
-4 and $-1 \mathrm{hr}$ contribute to the stenotele population at $96 \mathrm{hr}$. As the labeling period is extended to progressively later times, more precursors enter $\mathrm{S}$ phase, become labeled, and differentiate stenoteles. Thus the increase in the number of labeled stenoteles is a measure of the kinetics with which stenotele precursors enter $\mathrm{S}$ phase. These kinetics define the G1/S boundary of the precursor population. Since precursors labeled before the start of regeneration contribute to the stenotele population, it is clear that stenotele commitment occurs after the beginning of $\mathrm{S}$ phase.

\section{DISCUSSION}

In a previous report we showed that commitment to stenotele and desmoneme differentiation occurs during the terminal cell cycle of nests of four and eight precursors (Fujisawa and David, 1981). In the present experiments we have used an assay for autonomous differentiation to quantitate the appearance of committed stenotele precursors during regeneration of isolated distal regions of the body column. In the same tissue we have determined the G1/S boundary and S/G2 boundary of the precursor population using standard cell cycle techniques. Comparing the two sets of data (Fig. 6) indicates that the increase in stenotele commitment occurs near the S/G2 boundary of the precursor population. However, because of the 1- to 2-hr uncertainty in the position of the commitment curve (see Results), we cannot at present determine whether commitment occurs in late S phase or in early G2. The duration of the $S$ phase derived from these experiments is about $11 \mathrm{hr}$ and the time from the end of $\mathrm{S}$ to differentiated capsules is about $73 \mathrm{hr}$. Both estimates are in good agreement with previous observations obtained

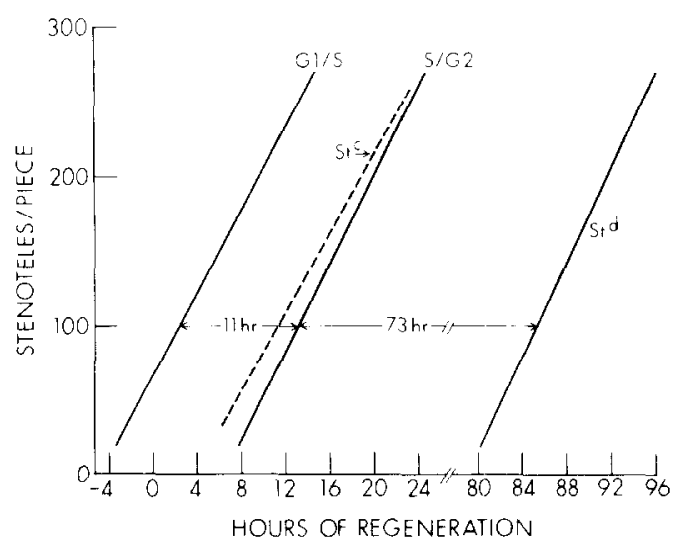

FIG. 6. Schematic representation of cell cycle of stenotele precursors during regeneration. Data from Figs. 1, 4, and 5 were combined. $\mathrm{St}^{\mathrm{d}}$, appearance of newly differentiated stenoteles during regeneration (Fig. 1); $\mathrm{St}^{\mathrm{c}}$, appearance of committed stenotele precursors during regeneration (Fig. 1). G1/S boundary (Fig. 5). S/G2 boundary (Fig. 4). 
with different techniques (David and Campbell, 1972; Campbell and David, 1974; David and Gierer, 1974).

The localization of stenotele commitment close to the S/G2 boundary is interesting in view of observations localizing nerve commitment in Hydra to mid to late S phase (Berking, 1979; Venugopal and David, 1981; Yaross, Baca, Chow and Bode, 1982). Both events involve a clear choice between different pathways of cytodifferentiation although one occurs at the level of the interstitial stem cell while the other occurs in nests of cells committed to nematocyte differentiation but not committed as to type of nematocyte capsule. Whether this coincidence of commitment events and $\mathrm{S}$ phase has mechanistic significance is not yet clear. Nevertheless, it should be noted that "commitment" events in erythroblast differentiation (Harrison, 1976; Gusella et al., 1976) or myoblast differentiation (Nadal-Ginard, 1978), which have been localized to G1, may not be directly comparable to our findings since these events do not represent choices between pathways of differentiation but rather the choice to express a particular differentiation pathway for which the cells are already programmed.

This research was supported by grants from the NIH (GM11301) and the NSF (77-25426). C.N.D. is recipient of a Career Development Award (FRA-132) from the American Cancer Society. T.F. is recipient of a fellowship from the Japanese Ministry of Education.

\section{REFERENCES}

BERKING, S. (1979). Control of nerve cell formation from multipotent stem cells in Hydra. J. Cell Sci. 40, 193-205.

BoDE, H. R., and SMITH, G. S. (1977). Regulation of interstitial cell differentiation in Hydra attenuala. II. Correlation of the axial posi- tion of the interstitial cell with nematocyte differentiation. Wilhelm Roux's Arch. Entwicklungsmech. Org. 181, 203-213.

CAMPBELl, R. D., and DAvid, C. N. (1974). Cell cycle kineties and development of Hydra attenuata. II. Interstitial cells. J. Cell Sci. 16, 349-358.

DAVID, C. N. (1973). A quantitative method for maceration of Hydra tissue. Wilhelm Roux's Arch. Entwicklungsmech. Org. 171, 259-268.

Davin, C. N., and CAMPBELL, R. D. (1972). Cell cycle kinetics and development of Hydra attenuata. I. Epithelial cells. J. Cell Sci. 11, $557-568$.

David, C. N., and Challoner, D. (1974). Distribution of interstitial cells and differentiating nematocytes in nests in Hydra attenuata. Amor. Zool 14, 537-512.

DAvID, C. N., and GIERER, A. (1974). Cell cycle kinetics and development of Hydra attenuata. III. Nerve and nematocyte differentiation. J. Cell Sci. 16, 359-375.

Fujisawa, T., and David, C. N. (1981). Commitment during nematocyte differentiation in $H y d r a . J$. Cell Sci. 48, 207-222.

Gusella, J., Geller, R., Clarke, B., Weeks, V., and Housman, D. (1976). Commitment to erythroid differentiation by Friend erythroleukemia cells: a stochastic analysis. Cell 9, 221-229.

HARRISON, P. R. (1976). Analysis of erythropoiesis at the molecular level. Nature (London) 262, 353-356.

LEHN, H. (1951). Teilungsfolgen und determination von I-zellen für die Cnidenbildung bei Hydra. Z. Naturforsch. 6b, 388-391.

NADEL-GINARD, B. (1978). Commitment, fusion and biochemical differentiation of a myogenic cell line in the absence of DNA synthesis. Cell 15, 855-864.

RICH, F, and TARDENT, P. (1969). Untersuchung zur NematocytenDifferenzierung bei Hydra attenuata. Rev. Suisse Zool. 76, 779-789.

SlautTERBACK, D. B., and FAwCETT, D. W. (1959). The development of the cnidoblasts of Hydra. An electron microscope study of cell differentiation. J. Biophys. Biochem. Cytol. 5, 441-450.

VENUGOPAL, G., and DAvid, C. N. (1981). Nerve commitment in Hydra. II. Localization of commitment in S-phase. Dev. Biol. 83, 361-365.

WILBY, O. K., and WEBSTER, G. (1970). Experimental studies on axial polarity in Hydra. J. Embryol. Exp. Morphol. 24, 595-613.

Yaross, M. S., BACA, B. A., CHOW, M. H., AND BODE, H. R. (1982). Commitment of Hydra interstitial cells to nerve cell differentiation vecurs by late S-phase. Dev. Biol 89, 425-436. 